\title{
A CASE REPORT OF AN ADULT MALE PATIENT WITH AVOIDANT/RESTRICTIVE FOOD INTAKE DISORDER TREATED WITH CBT
}

\author{
Matteo Aloi ${ }^{1}$, Flora Sinopoli ${ }^{2}$ \& Cristina Segura-Garcia ${ }^{1}$ \\ ${ }^{I}$ Department of Health Sciences, University Magna Graecia of Catanzaro, Catanzaro, Italy \\ ${ }^{2}$ Dietetic Service, University Hospital Mater Domini, Catanzaro, Italy
}

received: 11.7.2017;

revised: 16.11.2017;

accepted: 17.1.2018

* * * * *

\section{INTRODUCTION}

Avoidant/Restrictive Food Intake Disorder (ARFID) is a new diagnosis within the Feeding and Eating Disorders in DSM-5 (American Psychiatric Association 2013). It has replaced the Feeding Disorder of Infancy and Early Childhood (FDIEC) included in the DSM-IVTR. ARFID diagnosis is intended to gather individuals who meet criteria for the previous FDIEC, but also to collect other patients with clinically significant eating problems who were formerly defined as Eating Disorder Not Otherwise Specified (EDNOS).

ARFID symptoms typically become evident in infancy or childhood, but they may also present or persist during adulthood. People who fulfill the criteria have developed some kind of problem with eating (or for very young children, a problem with feeding) or food. As a result, the person is not able to take the enough nutrition or energy intake through diet and may end up losing weight, so some patients might need supplements to get adequate feeding. Younger kids might not gain weight or grow as expected (Kohn 2016).

Different types of eating behaviors might warrant an ARFID diagnosis, e.g. avoiding foods on the base of sensory characteristics like color or texture, difficulty digesting certain foods, eating only very small portions, having no appetite or lacking interest in food or eating, or being afraid to eat after a frightening episode of choking or vomiting. Many individuals with ARFID may have problems at school or work because of their eating struggles such as avoiding work lunches, not getting schoolwork done because of the time it takes them to eat, or even avoiding meeting friends or family during social events where food is present.

One of the clinical features of ARFID as described in DSM-5 (i.e. concern about aversive consequences of eating) may overlap with Orthorexia Nervosa (ON) (Dunn \& Bratman 2015). ON is characterized by the obsessive focus on "healthy" eating, as defined by a dietary theory or set of beliefs whose specific details may vary and it is marked by exaggerated emotional distress related to food choices perceived as unhealthy.
There are few studies in the literature about ARFID and most of them have been done with children and adolescents (Mammel \& Ornstein 2015, Norris et al. 2014). Besides, recently, we found studies that describe this disorder in adult patients, predominantly women (Nakai et al. 2016, 2017). Instead we present the case of an adult male ARFID from the first psychiatric evaluation until a follow-up of 6 months after treatment. This case report aims to describe if a Cognitive Behavioral Therapy (CBT) approach with the family involvement is effective in the treatment of ARFID in adulthood.

\section{CASE ILLUSTRATION: PRESENTATION}

AA, a 24-year-old male, sought help at an outpatient ED Unit in a university Mental Health service. He manifested subjective distress and growing relationship difficulties consequential to his dysfunctional eating behavior that he dated back at the age of 2 years.

AA complained anticipatory anxiety about sharing meals with colleagues at work; therefore he manifested avoidant behaviors when he was invited for lunch or dinner resulting in social withdrawal. He felt constrained to confess this problem to his headman to avoid the business lunches.

He admitted that his nutrition was poor and was well motivated to address his behavior. The onset was not clearly connected to particular life events (e.g. episodes of choking or vomiting) but he stated "everything changed when I put the teeth".

AA, who was the eldest of two brothers and two sisters, lived with his family; none of them had any current or past significant clinical or mental disorder. During childhood, whenever he was repeatedly visited by the pediatrician and other specialized physicians "because he did not eat", was anemic and underweight, doctors reassured his parents saying "do not insist because he will unlock on his own" and discarded any kind of neurological, structural or congenital disorder. He always avoided meat (except chicken that started 
eating two years ago), fish, legumes, vegetables (except lettuce) and fruits on the basis of anticipatory unpleasant perceptual experience ("the smell and the color disgust me and I vomit if I try to eat them"). His typical dietary day intake was as follows: breakfast with coffee, milk and biscuits or sweet snacks at home; for lunch and dinner he ate either "margherita pizza" or pasta with so much bread crumbs and oil, or baked rice/pasta, or all kind of cooked potatoes and sweets.

The physical exploration gave no data of interest; the patient was slightly overweight (i.e. weight: $72 \mathrm{~kg}$; height: $1.68 \mathrm{~m}$ ) (Table 1) due to mainly consuming carbohydrates and multivitamin and mineral integrators. Blood tests revealed that he was a $\beta$-thalassemia carrier. Bioelectrical Impedance Analysis (BIA) showed a good nutritional status. The psychological evaluation confirmed an avoidant personality trait through the Temperament and Character Inventory-Revised. Only high Maturity Fears were evident at the Eating Disorder Inventory 2 (EDI-2). The ORTO-15 score was 35, thus indicating the positivity for Orthorexia Nervosa. AA described himself as an euthymic person, hard worker, "precise, orderly and reliable" and appeared calm, little assertive, quiet and introverted; he was submissive and passive in social relationships because he could not tolerate the threat of punishment, pain or social disapproval, defining himself as rigid and passive.

Individuals with Anorexia Nervosa (AN) or Bulimia Nervosa $(\mathrm{BN})$ show body image distortion and significant concerns about their weight. Instead patients with ARFID typically do not fear weight gain and do not have a distorted body image. AA showed no body image concern or weight phobia; in fact he had normal scores in Body Dissatisfaction (BD) and Drive for Thinness (DT). So AN and BN diagnosis were rejected. On the other hand, the patient did not show any concern about healthy eating or about the possible harmful consequences of not following any specific dietary pattern, nor did he declare to worry about any damaging result for his health in relation to the foods that he dodged. He just avoided eating foods because of their unpleasant sensory characteristics (Dunn \& Bratman, 2015). So, the diagnosis of ON, even if his ORTO-15 score indicated the opposite, was discarded.

Anxiety disorders were also discarded as AA had never experienced situations that may had lead to choking or vomiting that could have been considered the triggers for the fear or the avoidance required for these diagnosis. Then again obsessive-compulsive disorder was rejected because the patient's thought were not intrusive or time consuming and he did not show ritualized eating behaviors. In a similar way schizophrenia spectrum disorders were not considered as possible diagnosis because delusional beliefs were not present.

Considering all above described, the psychiatrist made the diagnosis of ARFID and proposed a cognitivebehavioral therapy (CBT) approach.
Table 1. Tests scores and lab parameters in relation to treatment

\begin{tabular}{|c|c|c|c|}
\hline & $\mathrm{t}_{0}$ & $\mathrm{t}_{1}$ & $\mathrm{t}_{2}$ \\
\hline \multicolumn{4}{|l|}{ EDI-2 } \\
\hline Drive for Thinness & 0 & 0 & 0 \\
\hline Bulimia & 0 & 0 & 0 \\
\hline Body Dissatisfaction & 3 & 6 & 5 \\
\hline Ineffectiveness & 0 & 0 & 1 \\
\hline Perfectionism & 4 & 4 & 2 \\
\hline Interpersonal Distrust & 3 & 1 & 1 \\
\hline Interoceptive Awareness & 3 & 0 & 0 \\
\hline Maturity Fears & 9 & 1 & 2 \\
\hline Ascetism & 5 & 4 & 2 \\
\hline Impulse Regulation & 3 & 1 & 2 \\
\hline Social Insecurity & 1 & 2 & 2 \\
\hline ORTO-15 & 35 & 31 & 39 \\
\hline Body Mass Index $\left(\mathrm{kg} / \mathrm{m}^{2}\right)$ & 25.5 & 26.9 & 26.6 \\
\hline White blood cells $\left(\mathrm{x} 10^{3} / \mu \mathrm{L}\right)$ & 9.1 & & 10.0 \\
\hline Red blood cells (x 10\%/ $\mu \mathrm{L})$ & 5.46 & & 5.65 \\
\hline Hemoglobin $(\mathrm{g} / \mathrm{dL})$ & 11.3 & & 11.7 \\
\hline Platelets $\left(\times 10^{3} / \mu \mathrm{L}\right)$ & 263 & & 292 \\
\hline Hematocrit (\%) & 35.1 & & 36.3 \\
\hline Mean Corpuscular Value (fL) & 64.4 & & 64.3 \\
\hline Mean Corpuscular Hemoglobin (pg) & 20.8 & & 20.8 \\
\hline Mean Platelet Value (fL) & 11.6 & & 11.5 \\
\hline Glycaemia (mg/dL) & 105 & & 94 \\
\hline Creatinine (mg/dL) & 0.79 & & 0.86 \\
\hline Cholesterol total (mg/dL) & 146 & & 129 \\
\hline GOT (IU/L) & 20 & & 18 \\
\hline GPT (IU/L) & 28 & & 23 \\
\hline GGT (IU/L) & 24 & & 22 \\
\hline Blood urea nitrogen $(\mathrm{mg} / \mathrm{dL})$ & 24 & & 35 \\
\hline \multicolumn{4}{|l|}{ Bioelectrical Impedance Analysis } \\
\hline Phase angle $\left(^{\circ}\right)$ & 6.2 & & 6.4 \\
\hline Total Body Water (L) & 44.0 & & 44.3 \\
\hline Extracellular Water (L) & 19.7 & & 19.4 \\
\hline Body Cell Mass $(\mathrm{kg})$ & 31.2 & & 31.8 \\
\hline
\end{tabular}

Psychologist defined and settled treatment goals with the patient and therapy was carried once a week in onehour sessions for six months (Figure 1). The program, which was carried out with a single therapist with special training in the field of EDs, started with four psycho-education sessions. The mother and subsequently all family members were involved during the following three sessions. Then the patient completed the weekly CBT program individually.

Self-monitoring with the compilation of a food diary, behavioral exposures (e.g. buying, cooking and eating new meals during sessions), cognitive restructuring and techniques to manage anxiety were used in order to achieve the main goals (i.e. to manage stressful situations linked to job and social relationships and to achieve autonomy from family). The patient made a list of foods that he would accept to taste; he was offered one of them at each session. Then he described and 
noted the smell, texture and sensations that he perceived when testing each new food. He was given the chance to chew and eventually spit them out if disgusted.

The family displayed some dysfunctional dynamics: both sisters had high expressed emotions with hostile and denigrating communication towards AA's initial attempts to introduce new foods; the mother appeared low compliant and unable to cope with the initial signs of food rejection; the younger brother expressed strong opposition ("disengagement" according to patient) and never reached the family-sessions. The father, who had separated from the mother the year before, also denied his involvement in the family-sessions but was interested about the progress of treatment.

A possible correlation of AA's disorder could be referred to his maturity fears; in that sense his "eating behavior" could be related to the desire of remaining under the protection of the family. Indeed the patient confirmed the impairment after his parents' separation that he linked to the burden of his new role as the "senior male" in the family. The patient gained the awareness of this fact during the psychotherapy. AA reacted positively when these family dynamics were addressed during the sessions, displaying good motivation and therapeutic alliance (e.g. the constant presence and punctuality at the interviews).

The exposure therapy was very effective because the gradual exposure to new foods allowed expanding his culinary options through a desensitization process within a neutral environment free of anxiety. At the end of CBT, six months later, AA had introduced many new foods in his diet like fish (e.g. swordfish, cod-fish), meat (e.g. chicken, turkey, beef, pork), vegetables (e.g. tomatoes, carrots) and fruits (e.g. apple, banana, pear, strawberry) that allowed him broader social relationships.

Tests after $6\left(\mathrm{t}_{1}\right)$ and $12\left(\mathrm{t}_{2}\right)$ months confirmed the clinical improvement. EDI-2 scores were reduced in nearly every dimension from $t_{0}$ to $t_{2}$. The biggest drop regarded Maturity Fears. The slight increment of BD was parallel to weight gain. Health status showed an improvement as demonstrated by BIA and blood lab parameters (Table 1).

Interestingly, the patient's ORTO-15 scores were respectively $31\left(\mathrm{t}_{1}\right)$ and $39\left(\mathrm{t}_{2}\right)$, so he resulted even more positive to ON during the CBT psychotherapy. Another research previously described the increased rate of $\mathrm{ON}$ among ED patients after treatment (Segura Garcia et al. 2015). Two explanations can be given to this result: a) this orthorexic score could be an iatrogenic-like side effect of CBT, but according to clinical interview was not confirmed, b) the result was a false positive: a recent research has highlighted the lack of statistical power of the ORTO-15 (Missbach et al. 2015).

At $\mathrm{t} 2$, another six months after the end of the CBT program, the patient appeared calmer, continued his discovery of new flavors, managed to overcome the failure at the first approach with new meals and feels supported by his family.

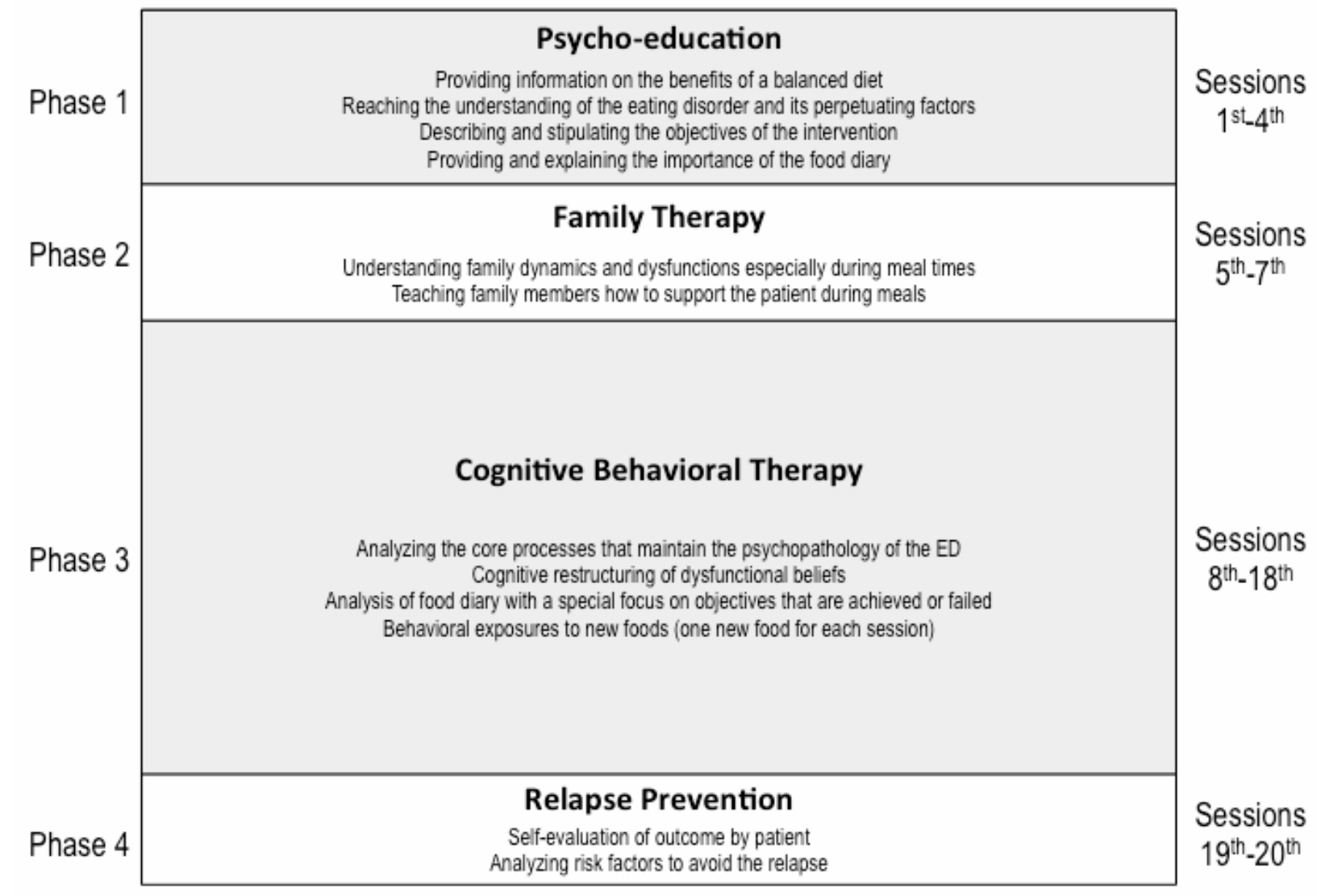

Figure 1. Description of the psychotherapeutic intervention 


\section{CONCLUSION}

Although ARFID is an ED commonly diagnosed in childhood this case report demonstrates that it can present in adult age. CBT, that has proved its effectiveness in the treatment of other EDs (e.g. BN and Binge Eating Disorder), seems also effective in treating ARFID. Although research is just beginning, we do believe that behavioral interventions, such as exposure therapy, together with the involvement of family may be useful to treat patients with ARFID.

\section{References}

1. American Psychiatric Association: Diagnostic and statistical manual of mental disorders (5th ed.). APA, Washington, DC, 2013

2. Dunn TM, Bratman S: On orthorexia nervosa: A review of the literature and proposed diagnostic criteria. Eat Behav 2015; 21:11-17

3. Kohn JB: What Is ARFID? J Acad Nutr Diet 2016; 116:1872

4. Mammel KA, Ornstein RM: Avoidant/restrictive food intake disorder: a new eating disorder diagnosis in the diagnostic and statistical manual 5. Curr Opin Pediatr 2017; doi: 10.1097/MOP.0000000000000507

5. Nakai $Y$, Nin $K$, Noma $S$, Teramukai $S$, Wonderlich $S A$ : Characteristics of Avoidant/Restrictive Food Intake Disorder in a Cohort of Adult Patients. Eur Eat Disord Rev 2016; $24: 528-530$
Acknowledgements: None.

Conflict of interest: None to declare.

\section{Contribution of individual authors:}

Matteo Aloi and Cristina Segura Garcia: Design of the study and interpretation of data;

Matteo Aloi: Clinical evaluations and psychotherapeutic treatment;

Flora Sinopoli: collected nutritional data.

6. Nakai $Y$, Nin $K$, Noma $S$, Hamagaki $S$, Takagi $R$, Teramukai $S$, Wonderlich $S A$ : Clinical presentation and outcome of avoidant/restrictive food intake disorder in a Japanese sample. Eat Behav 2017; 24:49-53

7. Norris ML, Robinson A, Obeid N, Harrison M, Spettigue $W$, Henderson $K$ : Exploring avoidant/restrictive food intake disorder in eating disordered patients: a descriptive study. Int J Eat Disord 2014; 47:495-499

8. Missbach B, Hinterbuchinger B, Dreiseitl V, Zellhofer S, Kurz C, König J: When Eating Right, Is Measured Wrong! $A$ Validation and Critical Examination of the ORTO-15 Questionnaire in German. PLoS One 2015; 10:e0135772

9. Segura-Garcia $C$, Ramacciotti $C$, Rania $M$, Aloi $M$, Caroleo M, Bruni A, Gazzarrini D, Sinopoli F, De Fazio $P$ : The prevalence of orthorexia nervosa among eating disorder patients after treatment. Eat Weight Disord 2015; 20:161-166

Correspondence:

Cristina Segura-García, $M D, P h D$

Department of Health Sciences, University Magna Graecia of Catanzaro

Viale Europa, 88100-Catanzaro, Italy

E-mail: segura@unicz.it 